\title{
Validation of the Polish version of the Movement Disorder Society-Unified Parkinson's Disease Rating Scale (MDS-UPDRS)
}

Joanna Siuda $^{1 \star}$, Magdalena Boczarska-Jedynak ${ }^{2 \star}$, Sławomir Budrewicz ${ }^{3}$, Jarosław Dulski ${ }^{4,5}$, Monika Figura $^{6}$, Urszula Fiszer ${ }^{7}$, Agata Gajos ${ }^{8}$, Agnieszka-Gorzkowska ${ }^{9}$, Ewa Koziorowska-Gawron ${ }^{3}$, Dariusz Koziorowski ${ }^{6}$, Anna Krygowska-Wajs ${ }^{10}$, Monika Rudzińska-Bar ${ }^{11}$, Jarosław Sławek ${ }^{4,5}$, Xuehan Ren ${ }^{12}$, Sheng Luo ${ }^{13}$, Pablo Martinez-Martin ${ }^{14}$, Glenn Stebbins ${ }^{15}$, Christopher G. Goetz ${ }^{15}$, Grzegorz Opala ${ }^{1}$, and the MDS-UPDRS Polish Validation Task Force ${ }^{\star *}$

${ }^{1}$ Department of Neurology, Faculty of Medical Sciences in Katowice, Medical University of Silesia, Katowice, Poland

${ }^{2}$ Department of Neurology and Regenerative Medicine, Dr Boczarska-Jedynak Health Institute, Oświęcim, Poland

${ }^{3}$ Department of Neurology, Wroclaw Medical University, Wroclaw, Poland

${ }^{4}$ Department of Neurological-Psychiatric Nursing, Medical University of Gdansk, Gdansk, Poland

${ }^{5}$ Neurology and Stroke Department, St. Adalbert Hospital, Gdańsk, Poland

${ }^{6}$ Department of Neurology, Faculty of Health Sciences, Medical University of Warsaw, Warsaw, Poland

${ }^{7}$ Department of Neurology and Epileptology, Centre of Postgraduate Medical Education, Orłowski Hospital, Warsaw, Poland

${ }^{8}$ Department of Extrapyramidal Diseases, Medical University of Łódź, Łódź, Poland

${ }^{9}$ Department of Neurorehabilitation, Faculty of Medical Sciences in Katowice, Medical University of Silesia, Katowice, Poland

${ }^{10}$ Department of Neurology, Jagiellonian University, Collegium Medicum, Krakow, Poland

${ }^{11}$ Department of Neurology, Faculty of Medicine and Health Sciences, Andrzej Frycz Modrzewski Krakow University, Krakow, Poland

${ }^{12}$ Department of Biostatistics, Gilead Sciences, Inc., Foster City, California, USA

${ }^{13}$ Department of Biostatistics \& Bioinformatics, Duke University, Durham, North Carolina, USA

${ }^{14}$ National Centre of Epidemiology and CIBERNED, Carlos III Institute of Health, Madrid, Spain

${ }^{15}$ Department of Neurological Sciences, Rush University Medical Centre, Chicago, Illinois, USA

${ }^{16}$ Department of Neurology, The John Paul II Specialist Hospital in Kraków, Poland

\section{ABSTRACT}

Introduction. In 2008, the Movement Disorders Society (MDS) published a new Unified Parkinson's Disease Rating Scale (MDS-UPDRS) as the official benchmark scale for Parkinson's Disease (PD). We have translated and validated the Polish version of the MDS-UPDRS, explored its dimensionality, and compared it to the original English one.

Methods. The MDS-UPDRS was translated into Polish by a team of Polish investigators led by JS and GO. The back-translation was completed by colleagues fluent in both languages (Polish and English) who were not involved in the original translation, and was reviewed by members of the MDS Rating Scales Programme. Then the translated version of the MDS-UPDRS underwent cognitive pretesting, and the translation was modified based on the results. The final translation was approved as the Official Working Document of the MDS-UPDRS Polish version, and was tested on 355 Polish PD patients recruited at movement disorders centres all over Poland (at Katowice, Gdańsk, Łódź, Warsaw, Wrocław, and Kraków). Confirmatory and explanatory factor analyses were applied to determine whether the factor structure of the English version could be confirmed in the Polish version.

* translation/back-translation team members

** Task Force members in alphabetical order: Andrzej Bogucki ${ }^{8}$, Piotr Janik ${ }^{6}$, Magdalena Koszewicz $^{3}$, Marta Leńska-Mieciek, Małgorzata Michałowska ${ }^{7}$, Marta Piaścik-Gromada ${ }^{7}$, Katarzyna Potasz-Kulikowska ${ }^{10}$, Marek Śmiłowski ${ }^{1 \star}$, Anna Wasielewska ${ }^{16}$, Magdalena Wójcik-Pędziwiatr ${ }^{11}$

Address for correspondence: Joanna Siuda, Department of Neurology, Faculty of Medical Sciences in Katowice, Medical University of Silesia, Katowice, Poland, email: jsiuda@sum.edu.pl 
Results. The Polish version of the MDS-UPDRS showed satisfactory clinimetric properties. The internal consistency of the Polish version was satisfactory. In the confirmatory factor analysis, all four parts had greater than 0.90 comparative fit index (CFI) compared to the original English MDS-UPDRS. Explanatory factor analysis suggested that the Polish version differed from the English version only within an acceptable range.

Conclusions and clinical implications: The Polish version of the MDS-UPDRS meets the requirements to be designated as the Official Polish Version of the MDS-UPDRS, and is available on the MDS web page. We strongly recommend using the MDS-UPDRS instead of the UPDRS for research purposes and in everyday clinical practice.

Key words: MDS-UPDRS, Parkinson's Disease, rating scale, validation, translation

(Neurol Neurochir Pol 2020; 54 (5): 417-425)

\section{Introduction}

Parkinson's Disease (PD) is a progressive, incurable, neurodegenerative disease presenting with motor and non-motor symptoms. There is no ideal biomarker to determine disease severity, and many different clinical, laboratory, and neuroimaging markers are used [1-3].

The 'gold standard' clinical rating scale for $\mathrm{PD}$, and the one most commonly used, is the Unified Parkinson's Disease Rating Scale (UPDRS) introduced in the 1980s [4], with its revised version known as MDS-UPDRS available from 2008 [5].

The MDS-UPDRS is composed of four sections: Part I: Non-Motor Experiences of Daily Living, has six rater-based items and seven for patient self-assessment; Part II: Motor Experiences of Daily Living, with 13 patient-based items; Part III: Motor Examination, with 18 items (33 scores); and Part IV: Motor Complications, with six items covering dyskinesia and fluctuations assessment. Each item scores from 0 (normal) to 4 (severe) and total scores are obtained from the sum of the corresponding item scores for each part. Differing from the previous version, the MDS-UPDRS is completed by a mix of both clinician-rated sections (Part 1A, Part 3 and Part 4) and patient- and/or caregiver-completed sections (Part 1B and Part 2), without direct input from the rater.

The new version relies on more information coming directly from patient and caregiver, and so it is vital that they understand the questions, preferably in their mother tongue. For this reason, the MDS Rating Scales Programme sets a specific protocol to designate successful translation of non-English versions. We aimed to translate and validate a Polish version of the MDS-UPDRS scale, and to compare it to the original English language version. Herein, we present the scale translation and clinimetric testing results of the Polish version of the MDS-UPDRS.

\section{Clinical rationale for the study}

The MDS-UPDRS has become the 'gold standard' of clinical assessment of PD patients. Being an expanded patient/ /caregiver-reported questionnaire means that it relies more on subjective information from the patient and/or his or her caregiver. The presented validation of the Polish translation of the MDS-UPDRS that is consistent with the English language version, confirms its suitability for use, and guarantees obtaining results for research purposes and everyday clinical practice that are comparable across different languages.

\section{Material}

The participants were 355 PD patients recruited from the neurology departments in ten sites located in different parts of Poland (two in Katowice, two in Krakow, one in Wroclaw, three in Warsaw, one in Gdansk, and one in Lodz). At each site experienced Polish movement disorder specialists were recruited to examine Polish-speaking PD patients of all ages, and at all disease Hoehn \& Yahr stages.

\section{Methods}

\section{Translation of the MDS-UPDRS}

The MDS Rating Scales Programme has prepared a well-defined protocol, with objective criteria for translation and validation of non-English versions of the MDS-UPDRS in order to have an "official MDS translation" in a foreign language. The process of developing an officially approved translation of the MDS-UPDRS involves four steps: (1) translation and independent back-translation; (2) cognitive pretesting to establish that the translation is clear and comfortably administered by native-speaker raters and understood by native-speaker patients; (3) field testing in the native language using a large sample of PD patients; and (4) full clinimetric testing [6].

The Polish version validation was performed as follows: firstly, the MDS-UPDRS was translated into Polish by a team of Polish-speakers who were fluent in English, and who were physicians and specialists in movement disorders. This team was led by Dr. Joanna Siuda, and Prof. Grzegorz Opala. It was then back-translated into English by colleagues fluent in English and Polish and who had not been involved in the original translation. Finally, it was reviewed by a team of American experts, led by Profs. Christopher Goetz and Glenn Stebbins, 
who had been involved in the development of the original English language version [7].

\section{Cognitive pretesting}

Cognitive pretesting is a qualitative approach to assessing instrument completion in terms of task difficulty for examiner and respondent, and respondent interest, attention span, discomfort, and comprehension [8]. Where differences were observed between the back-translated Polish version and the English version, items were selected for cognitive pretesting along with questions that were identified in cognitive testing of the English version. Questions included in cognitive pretesting were: Cognitive Impairment, Anxious Mood, Handwriting, Freezing, Hand Movements, Arising from Chair, Time Spent with Dyskinesia, and Functional Impairment of Dyskinesia. Based on the results of the initial cognitive pretesting, other round(s) of translation, back-translation and cognitive pretesting could be required. Once cognitive pretesting was completed and no problems had been noted, the final translation was obtained.

\section{Factor analysis}

M-plus Version 7.4 was used to do the primary confirmatory and secondary exploratory factor analyses, as the variables are categorical [9]. We used an unweighted least squares (ULS) approach to factor estimation that minimises the sum of squared differences between observed and estimated correlation matrices, not counting diagonal elements. To assist in interpretation of the factors, we used an orthogonal CF-VARIMAX rotation that constrains the factors to be uncorrelated.

The sample size for the translation study was based on the need for five subjects per item of the questionnaire in order to perform the statistical analysis [10]. Because there are 65 items on the MDS-UPDRS, a sample of at least 350 was required. Any participants with missing values within a Part were deleted from analysis of that Part only. Thus, the sample size from Part to Part could vary.

\section{Primary analysis}

For the primary analysis of the Polish data, we conducted a confirmatory factor analysis (CFA) to determine whether the factor structure for the English language MDS-UPDRS could be confirmed in data collected using the Polish translation $[11,12]$. This was the primary question of interest. The CFA was conducted separately for MDS-UPDRS Parts I to IV, with the Polish data constrained to fall into the factors defined in the English language data. We evaluated the CFA results based on the Comparative Fit Index (CFI). According to the protocol, to establish a successful translation and to designate that translation as an official MDS translation of the MDS-UPDRS, it is required that the CFI for each Part (I-IV) of the translated MDS-UPDRS be 0.90 or greater relative to the English language version. Mean and variance-adjusted weighted least square (WLSMV) estimator is used to confirm model fit.

\section{Secondary analysis}

As a secondary analysis, we conducted an exploratory factor analysis for the Polish version of the MDS-UPDRS Parts I-IV to explore the underlying factor structure without the constraint of a pre-specified factor structure. We used a SCREE plot to choose the number of factors to retain for each MDS-UPDRS Part. The subjective scree test [13] uses a scatter plot of eigenvalues plotted against their ranks with respect to magnitude, to extract as many factors as there are eigenvalues that fall before the last large drop (i.e. an 'elbow' shape) in the plot. Once the factors were chosen, an item was retained in a factor if the factor loading for that item was 0.40 or greater. To assist interpretation of the factors, an orthogonal CF-VARIMAX rotation was used which sets the factors to be uncorrelated.

\section{Ethics}

All patients gave written consent to participate. The anonymised patient data was transferred to the US team for analysis via a secure website. The programme for validation of the MDS-UPDRS Polish version was approved for all sites by the Ethics Committee of the Medical University of Silesia in Katowice (KNW/0022/KB/110/14).

\section{Results}

\section{Cognitive pretesting}

Ten patients with Parkinson's Disease and their examiners were interviewed using the type of structured interview format typical for cognitive pretesting. No problems were identified for the raters. One of the 10 patients interviewed had difficulty comprehending the term "lightheadedness", and another patient reported difficulty in reading the material for the self-report sections of the scale because the font was too small. No other patient-identified difficulties were noted. Slight modifications of the scale were recommended from this round of testing. Five patients completed a second round of cognitive pre-testing, and no difficulties were identified. The modified version of the scale was approved as the Official Working Draft of the Polish MDS-UPDRS for testing in a larger group of patients with PD.

\section{Study population characteristics}

The demographic characteristics of the sample are set out in Table 1. The sample included 355 Polish PD patients (mean age $64.0 \pm 9.5$ years, $56.9 \%$ males), with mean disease duration of $9.0 \pm 5.9$ years, who were examined using the MDS-UPDRS. They were all native Polish-speaking PD patients, white Caucasians, and the majority had at least secondary education. The English language and Polish language cohorts were similar in terms of age and duration of disease. All Hoehn \& Yahr stages (H\&Y) were represented, with the majority (299 subjects, $87.9 \%$ ) being stages II and III. The distribution of $\mathrm{H} \& \mathrm{Y}$ was significantly different between the two cohorts ( $\mathrm{p}$-value $<0.0001$ ). 
Table 1. Demographic characteristics of study population

\begin{tabular}{|c|c|c|c|c|c|c|c|c|c|c|c|}
\hline \multirow{2}{*}{$\begin{array}{c}\text { Study } \\
\text { cohort }\end{array}$} & \multirow{2}{*}{$\begin{array}{l}\text { Male* } \\
\text { N (\%) }\end{array}$} & \multirow{2}{*}{$\begin{array}{l}\begin{array}{c}\text { Age** } \\
\text { (years) }\end{array} \\
\text { Mean } \\
\text { + SD }\end{array}$} & \multirow{2}{*}{$\begin{array}{c}\text { Disease } \\
\text { duration } \\
\text { *** } \\
\text { (years) } \\
\text { Mean } \\
+ \text { SD }\end{array}$} & \multicolumn{5}{|c|}{$\begin{array}{c}\text { Hoehn\&Yahr**** } \\
\text { staging } \\
\text { N (\%) }\end{array}$} & \multicolumn{3}{|c|}{ Education $* * * * *$} \\
\hline & & & & I & II & III & IV & $\mathbf{v}$ & Vocational & Secondary & $\begin{array}{c}\text { University } \\
\text { degree }\end{array}$ \\
\hline $\begin{array}{l}\text { Polish } \\
(\mathrm{N}=355)\end{array}$ & $\begin{array}{c}169 \\
(56.9)\end{array}$ & $64.0 \pm 9.5$ & $9.0 \pm 5.9$ & $\begin{array}{c}18 \\
(5.3)\end{array}$ & $\begin{array}{c}148 \\
(43.5)\end{array}$ & $\begin{array}{c}151 \\
(44.4)\end{array}$ & $\begin{array}{c}19 \\
(5.6)\end{array}$ & $\begin{array}{c}4 \\
(1.2)\end{array}$ & 20 & 131 & 133 \\
\hline $\begin{array}{l}\text { English } \\
(\mathrm{N}=876)\end{array}$ & $\begin{array}{c}554 \\
(63.2)\end{array}$ & $\begin{array}{c}67.5 \pm \\
10.9\end{array}$ & $8.3 \pm 6.7$ & $\begin{array}{c}63 \\
(7.3)\end{array}$ & $\begin{array}{c}467 \\
(53.9)\end{array}$ & $\begin{array}{c}174 \\
(20.1)\end{array}$ & $\begin{array}{c}109 \\
(12.6)\end{array}$ & $\begin{array}{c}53 \\
(6.1)\end{array}$ & NA & NA & NA \\
\hline
\end{tabular}

Data available for $297^{*}, 257^{* *}, 197^{* * *}, 340^{* * * *}$, and $284^{* * * * *}$ subjects in Polish PD population; SD — standard deviation, NA — not available

\section{Primary analysis: Confirmatory Factor Analysis (CFA)}

Table 2 displays the CFA models for each MDS-UPDRS Part. For all four Parts of the Polish MDS-UPDRS, the CFI, compared to the English-language factor structure, was 0.90 or greater. This confirmed that the pre-specified English factor structure was confirmed in the Polish dataset.

\section{Secondary analysis: Explanatory Factor Analysis (EFA)}

EFA analysis for the Polish MDS-UPDRS dataset differed from the EFA of the English language dataset in some areas. From the SCREE plot we extracted: for Part I - two factors, for Part II - three components, for Part III - seven factors, and for Part IV - two factors. The SCREE plots are given in Figure 1. Table 3 shows the results of the Exploratory Factor Analysis for the English and Polish languages.

For Part I, in contrast to the English language version of the MDS-UPDRS, Cognitive impairment and Hallucinations and psychosis loaded on factor 2, instead of factor 1. For Part II, Speech loaded on factor 3 instead of factor 1, Handwriting and Doing hobbies and other activities loaded on factor 2, which originally loaded on factor 1 in the English language. Eating tasks loaded on factor 1 instead of factor 2. Dressing and Freezing loaded on both factor 1 and factor 2, Hygiene and Turning in bed loaded on factor 2, which originally loaded on factor 3 in the English language. In Part III, Rigidity, neck did not load on any of the factors. Nine of the 33 items loaded on different factors in the two scales, and eight items loaded on more than one factor. Most of the items that loaded on different factors in the two versions also had cross-loadings on multiple factors. In Part IV, Time spent with dyskinesia did not load on any of the factors. Time spent in the OFF state and Functional impact of dyskinesia loaded on both factor 1 and factor 2 in the Polish Scale, having originally loaded on factor 1 and factor 2 respectively in the English language.

\section{Discussion}

The widespread use of the MDS-UPDRS instead of its predecessor the UPDRS, which was, and in some non-English
Table 2. Confirmatory factor analysis, CFA model fit

\begin{tabular}{|c|c|}
\hline \multicolumn{2}{|c|}{$\begin{array}{l}\text { Part l: Non-motor aspects of experiences of daily living } \\
\text { (a 2-factor model)* }\end{array}$} \\
\hline Polish language & $\mathrm{CFI}=0.90, \mathrm{RMSEA}=0.10$ (355 patients) \\
\hline English language & $\mathrm{CFI}=0.96, \mathrm{RMSEA}=0.06$ (849 patients) \\
\hline \multicolumn{2}{|c|}{$\begin{array}{l}\text { Part Il: Motor aspects of experience of daily living (a 3-factor } \\
\text { model) }\end{array}$} \\
\hline Polish language & $\mathrm{CFI}=0.97, \mathrm{RMSEA}=0.09$ (355 patients) \\
\hline English language & $\mathrm{CFI}=0.97, \mathrm{RMSEA}=0.09$ (851 patients) \\
\hline \multicolumn{2}{|c|}{ Part III: Motor examination (a 7-factor model) } \\
\hline Polish language & $\mathrm{CFI}=0.95, \mathrm{RMSEA}=0.10$ (355 patients) \\
\hline English language & $\mathrm{CFI}=0.95, \mathrm{RMSEA}=0.07$ (801 patients) \\
\hline \multicolumn{2}{|c|}{ Part IV: Motor complications (a 2-factor model) } \\
\hline Polish language & $\mathrm{CFI}=1.00, \mathrm{RMSEA}=0.16$ (355 patients) \\
\hline English language & $\mathrm{CFI}=1.00, \mathrm{RMSEA}=0.04$ (848 patients) \\
\hline
\end{tabular}

$\mathrm{CFI}$ - comparative fit index, RMSEA - root mean square error of approximation;

*Dopamine Dysregulation Syndrome (DDS) was not included in this analysis as it did not load on any factor

speaking countries still is, the gold standard tool for the clinical assessment of PD patients, is to be recommended. The Movement Disorders Society Rating Scales Programme leads the global translation effort of different assessment scales including the MDS-UPDRS. Currently, this programme includes 19 nonEnglish validated editions of the MDS-UPDRS.

The overall factor structure of the Polish language version of MDS-UPDRS was consistent with that of the English language version, confirming its suitability for use. A few isolated item differences in factor loadings were identified in the EFA of the Polish version. These small differences included some loading on more than one factor, while others loaded on a different factor, compared to the English MDS-UPDRS.

For Part I, in contrast to the English language version of the MDS-UPDRS, Cognitive impairment and Hallucinations and psychosis loaded on factor 2 instead of factor 1 . This might be explained by the fact that cognitive impairment in Polish society is still not well recognised, especially among the elderly where it is treated as a normal component of the ageing process, and so goes underreported. 


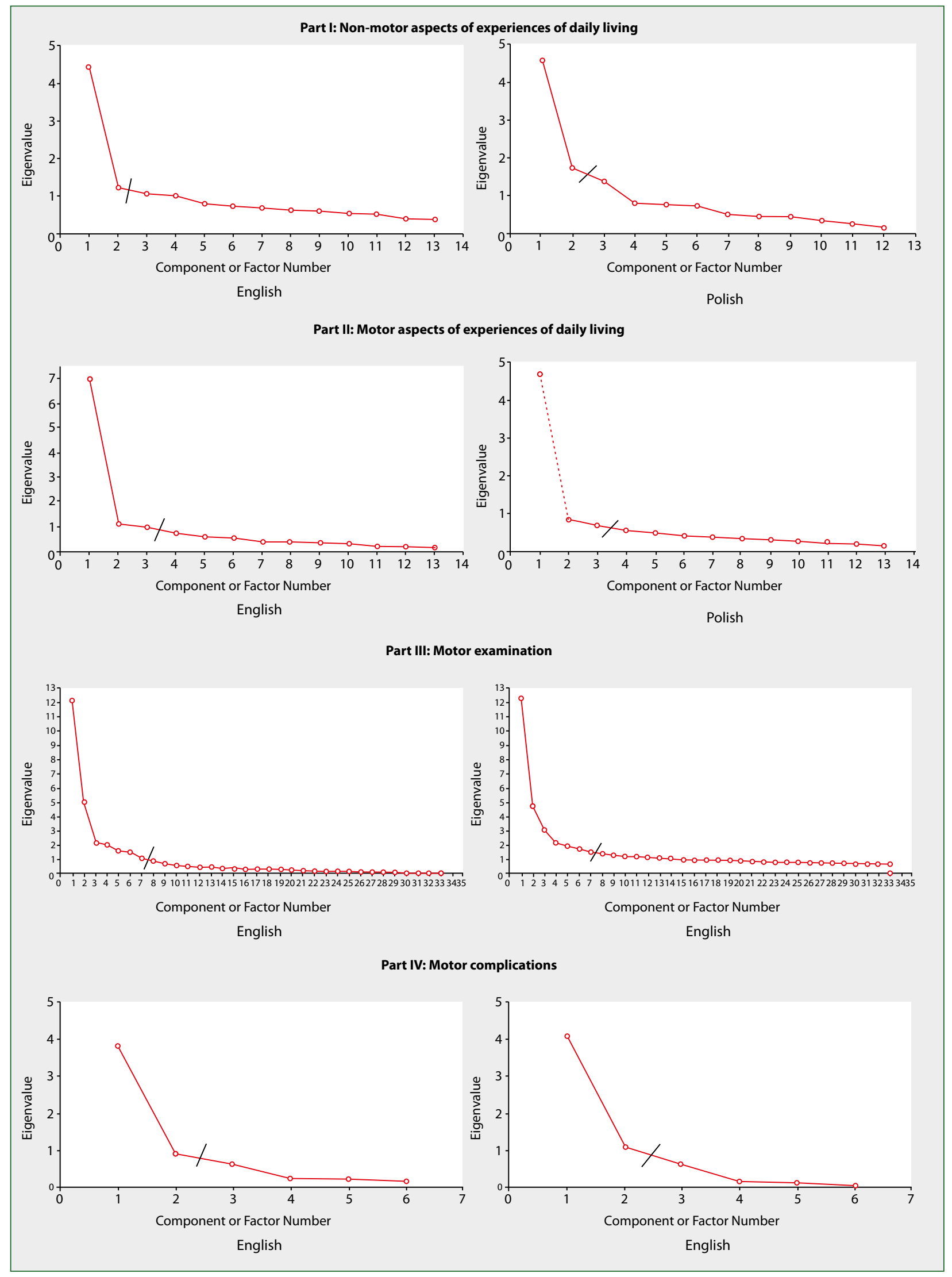

Figure 1. Scree plots for English and Polish exploratory factor analyses 
Table 3. Comparison of English language and Polish Exploratory Factor structures for Parts I-IV of MDS-UPDRS

\begin{tabular}{|c|c|c|c|}
\hline \multirow[t]{3}{*}{ Factor } & \multirow[t]{2}{*}{ Item } & \multicolumn{2}{|c|}{ Item factor loading } \\
\hline & & English* & Polish \\
\hline & \multicolumn{2}{|c|}{ Part I: Non-motor aspects of experiences of daily living } & \\
\hline \multirow[t]{10}{*}{ Factor 1} & Percent variance & 34.0 & 38.1 \\
\hline & Daytime sleepiness & 0.53 & 0.64 \\
\hline & Sleep problems & 0.35 & 0.55 \\
\hline & Cognitive impairment & 0.55 & $x x x x$ \\
\hline & Pain and other sensations & 0.43 & 0.67 \\
\hline & Hallucinations and psychosis & 0.56 & $x x x x$ \\
\hline & Urinary problems & 0.61 & 0.45 \\
\hline & Constipation problems & 0.46 & 0.53 \\
\hline & Lightheadedness on standing & 0.46 & 0.70 \\
\hline & Fatigue & 0.47 & 0.76 \\
\hline \multirow{6}{*}{ Factor 2} & Percent variance & 9.5 & 14.3 \\
\hline & Depressed mood & 0.81 & 0.84 \\
\hline & Anxious mood & 0.68 & 0.68 \\
\hline & Apathy & 0.55 & 0.83 \\
\hline & Hallucinations and psychosis & $x x x x$ & 0.44 \\
\hline & Cognitive impairment & $x x x x$ & 0.52 \\
\hline \multicolumn{4}{|c|}{ Part Il: Motor aspects of experiences of daily living } \\
\hline \multirow[t]{10}{*}{ Factor 1} & Percent variance & 53.10 & 50.73 \\
\hline & Speech & 0.79 & $x x x x$ \\
\hline & Saliva and drooling & 0.45 & 0.62 \\
\hline & Chewing and swallowing & 0.60 & 0.65 \\
\hline & Eating tasks & $x x x x$ & 0.56 \\
\hline & Dressing & $x x x x$ & 0.40 \\
\hline & Handwriting & 0.46 & $x x x x$ \\
\hline & Doing hobbies and other activities & 0.46 & $x x x x$ \\
\hline & Walking and balance & $x x x$ & $0.47^{* * *}$ \\
\hline & Freezing & $\mathrm{xxxx}$ & $0.41^{* * *}$ \\
\hline \multirow[t]{10}{*}{ Factor 2} & Percent variance & 8.70 & 8.90 \\
\hline & Eating tasks & 0.68 & $x x x x$ \\
\hline & Tremor & 0.43 & 0.56 \\
\hline & Handwriting & $x x x x$ & 0.94 \\
\hline & Hygiene & $x x x x$ & 0.75 \\
\hline & Doing hobbies and other activities & $\operatorname{xxxx}$ & 0.48 \\
\hline & Dressing & $x x x x$ & $0.49 * * *$ \\
\hline & Turning in bed & $x x x x$ & 0.59 \\
\hline & Walking and balance & $x x x$ & $0.48^{* * *}$ \\
\hline & Freezing & $x x x x$ & $0.46^{* * *}$ \\
\hline \multirow[t]{8}{*}{ Factor 3} & Percent variance & 7.70 & 7.33 \\
\hline & Speech & $\operatorname{xxxx}$ & 0.54 \\
\hline & Dressing & 0.64 & $x x x x$ \\
\hline & Hygiene & 0.65 & $x x x x$ \\
\hline & Turning in bed & 0.65 & $x X X X$ \\
\hline & Getting out of bed & 0.73 & 0.49 \\
\hline & Walking and balance & 0.82 & 0.52 \\
\hline & Freezing & 0.76 & $x X X X$ \\
\hline
\end{tabular}




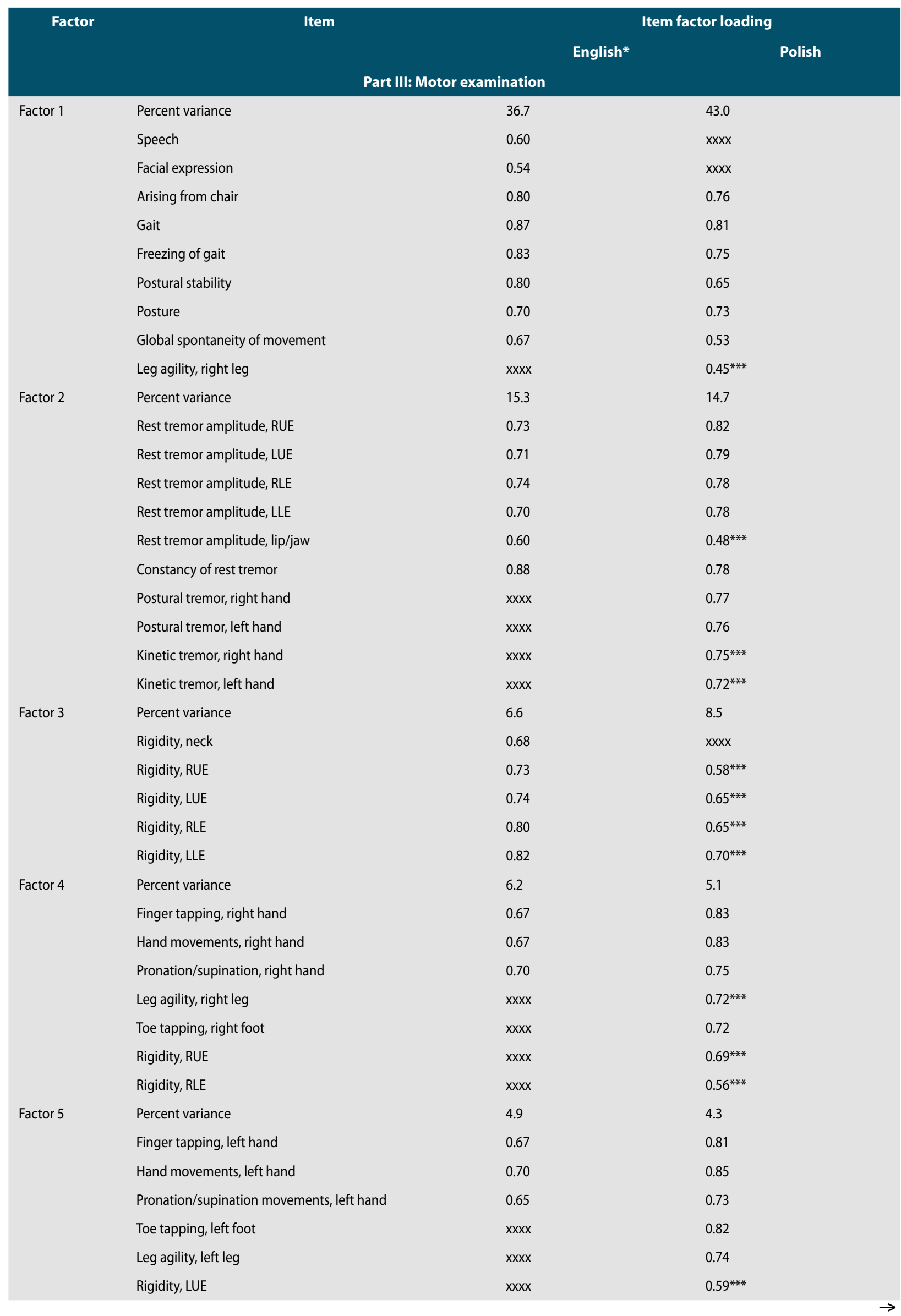




\begin{tabular}{|c|c|c|c|}
\hline \multirow[t]{2}{*}{ Factor } & \multirow[t]{2}{*}{ Item } & \multicolumn{2}{|c|}{ Item factor loading } \\
\hline & & English* & Polish \\
\hline \multicolumn{4}{|c|}{ Part IIl: Motor examination } \\
\hline \multirow{3}{*}{ Factor 6} & Rigidity, LLE & $x x x x$ & $0.47^{* * *}$ \\
\hline & Percent variance & 4.5 & 3.7 \\
\hline & Postural tremor, right hand & 0.66 & $x x x x$ \\
\hline \multirow{11}{*}{ Factor 7} & Postural tremor, left hand & 0.71 & $x x x x$ \\
\hline & Kinetic tremor, right hand & 0.81 & $0.44^{* * *}$ \\
\hline & Kinetic tremor, left hand & 0.81 & $0.44^{* * *}$ \\
\hline & Percent variance & 3.3 & 2.8 \\
\hline & Toe tapping, right foot & 0.65 & $x x x x$ \\
\hline & Toe tapping, left foot & 0.62 & $x x x x$ \\
\hline & Leg agility, right leg & 0.62 & $x x x x$ \\
\hline & Leg agility, left leg & 0.60 & $x x x x$ \\
\hline & Rest tremor amplitude, lip/jaw & $x x x x$ & $0.47^{* * *}$ \\
\hline & Speech & $x x x x$ & 0.67 \\
\hline & Facial expression & $x x x x$ & 0.76 \\
\hline \multicolumn{4}{|c|}{ Part IV: Motor complications } \\
\hline \multirow[t]{6}{*}{ Factor 1} & Percent variance & 63.9 & 67.4 \\
\hline & Time spent in the OFF state & 0.87 & $0.62^{* * *}$ \\
\hline & Functional impact of fluctuations & 0.84 & 0.91 \\
\hline & Complexity of motor fluctuations & 0.82 & 0.94 \\
\hline & Painful OFF state dystonia & 0.5 & 0.92 \\
\hline & Functional impact of dyskinesias & $x x x x$ & $0.48^{* * *}$ \\
\hline \multirow[t]{4}{*}{ Factor 2} & Percent variance & 15.6 & 17.6 \\
\hline & Time spent with dyskinesias & 0.71 & $x x x x$ \\
\hline & Functional impact of dyskinesias & 0.95 & 0.86 \\
\hline & Time spent in the OFF state & $x x x x$ & 0.68 \\
\hline
\end{tabular}

*Different version of M-Plus, and the factor loadings may vary slightly from published version; *** item load on more than one factor with factor loading $\geq 0.40$; $x x x x$ implies that the listed item did not load on the factor indicated

Moreover, PD patients may not recognise memory problems as part of a wide range of PD symptomatology, and thus do not report them. Another argument, raised by the team of MDS experts, is that a cognition item, assessed by a single screening question, has a weak correlation with the corresponding cognitive scales i.e. Addenbrooke's Cognitive Examination, ACE, Scales for Outcome of Parkinson's Diseasecognitive scale, SCOPA-COG, and Frontal Assessment Battery, FAB [14]. In regards to Hallucination and psychosis, rarely reported in the studied population (301 subjects; $84.8 \%$ reported no psychotic problems), the information mainly came from PD patients, and only rarely was the interview conducted in the presence of the patient's caregiver. Caregivers can be very good sources of information, especially when we are looking for information about non-motor symptoms of Parkinson's Disease, which possibly go unnoticed by patients.
For Part II, Speech loaded on factor 3 instead of factor 1 , and the majority of patients (231 subjects; $65.1 \%$ ) was assessed as either having no or minimal problems with speech. Handwriting and Doing hobbies and other activities loaded on factor 2, which originally loaded on factor 1 in the English language. Probably due to cultural differences, Polish PD patients tend to refer to hobbies and other activities done for pleasure in a more practical manner than do patients from English-speaking parts of the world. On the other hand, it has been demonstrated previously on a large multi-cultural and multi-language population that the MDS-UPDRS is effective in capturing parkinsonism, and is not highly influenced by gender, age, or race/ethnicity [15].

Nowadays, we tend to do less handwriting, and it is possible that patients do not pay much attention to this activity as long as they still have a legible signature. Eating tasks loaded 
on factor 1 instead of factor 2, Dressing loaded on both factor 1 and factor 2. Hygiene loaded on factor 2, which originally loaded on factor 3 in the English language. Walking and balance loaded on all three factors, unlike the English version that loaded only on factor 3. A possible explanation here is that independence is very important to our PD patients; they do not want anyone to help them unless it is absolutely necessary; and being able to dress, bathe, eat, or walk without assistance is for them a determinant of a good quality of everyday life.

In Part III, Rigidity, neck did not load on any of the factors, even though 219 (61.7\%) patients reported it. Resting tremor of the upper limbs loaded in factor 2 in both the English and the Polish versions, but Postural and kinetic tremor of both upper limbs loaded in factor 2 in the Polish version while being only factor 6 in the English language version. This can be explained by cultural differences between English- and Polish-speaking societies, by the fact that tremor is an easily recognisable symptom, and because for many of our patients it is embarrassing, especially in social situations. In the Polish version, Toe tapping and leg agility on the right side loaded in factor 4, and Toe tapping and leg agility on the left side loaded on factor 5, unlike the English version in which these items loaded only in factor 7 . Here again, bradykinesia in the lower limbs, probably causing gait problems, makes a patient less independent in their everyday life.

In Part IV, most items loaded on factor 1 in both language versions, underlining the importance of motor complications. There was one exception: in the Polish version Time spent with dyskinesia did not load on any of the factors, possibly because more than half (202 subjects, 56.9\%) of PD patients reported having no dyskinesia. On the other hand, Functional impact of dyskinesia and Time spent in the OFF state loaded on both factor 1 and factor 2 in the Polish version. This confirms that levodopa-induced motor complications significantly impact upon patient well-being, and are related to an impaired healthrelated quality of life (HRQoL) [16].

We are aware that this study has some limitations related to potential sample selection bias. The data comes from high reference neurology clinics specialising in movement disorders, and it does not represent the general PD patient population in Poland. In addition, the distribution of patients showed a predominance of patients in early Hoehn \& Yahr stages, with very few patients in advanced stages (only four subjects in H\&Y stage V; 1.12\%), and most patients (284 subjects, 80\%) were examined in the ON state. Furthermore, the assessment was based mainly on patients' responses, although caregivers may have valuable input into clinical evaluation, especially with regard to non-motor symptoms [17].

\section{Conclusions and clinical implications:}

The overall factor structure of the Polish version was consistent with that of the English version based on the high
CFIs for all four parts of the MDS-UPDRS in the confirmatory factor analysis (all CFI $\geq 0.90$ ). The Polish MDS-UPDRS was confirmed to share a common factor structure with the English version.

Therefore, the MDS-UPDRS Polish version was designated as the Official Polish version of the MDS-UPDRS and is available from the MDS website (https://www.movementdisorders. org/MDS-Files1/Education/Rating-Scales/MDS-UPDRS_Polish_Official_Translation_FINAL.pdf).

Acknowledgements: We would like to thank our patients for participating in this project. We are grateful to Shazia Ali and Nura Said for their assistance during the study preparation, in conducting and recapitulating the validation of the Polish translation of the MDS-UPDRS.

Funding: This publication was prepared without any external source of funding.

Conflicts of interest: The authors declare no conflict of interest.

Contributions: A detailed list of the individual contributions of each author is attached to the 'Author statement' document. All authors have approved the final version of this manuscript.

\section{References}

1. Emamzadeh FN, Surguchov A. Parkinson's Disease: Biomarkers, Treatment, and Risk Factors. Front Neurosci. 2018; 12: 612, doi: 10.3389/fnins.2018.00612, indexed in Pubmed: 30214392.

2. Barc K, Kuźma-Kozakiewicz M. Positron emission tomography neuroimaging in neurodegenerative diseases: Alzheimer's disease, Parkinson's disease, and amyotrophic lateral sclerosis. Neurol Neurochir Pol. 2019; 53(2): 99-112, doi: 10.5603/PJNNS.a2019.0013, indexed in Pubmed: 30855701.

3. Malec-Litwinowicz M, Plewka A, Plewka D, et al. The relation between plasma $\alpha$-synuclein level and clinical symptoms or signs of Parkinson's disease. Neurol Neurochir Pol. 2018; 52(2): 243-251, doi: 10.1016/j.pjnns.2017.11.009, indexed in Pubmed: 29342421.

4. Fahn S, Elton RL, Members of, et al. Members of the UPDRS Develoment Committee. Unified Parkinson's Disease rating scale. In:, , , , editors. Recent develoments in Parkinson's disease, vol. 2. Florham Park, NJ: Macmillan Health Care Information; 1987. : 153-164.

5. Goetz CG, Tilley BC, Shaftman SR, et al. Movement Disorder Society UPDRS Revision Task Force. Movement Disorder Society-sponsored revision of the Unified Parkinson's Disease Rating Scale (MDS-UPDRS): scale presentation and clinimetric testing results. Mov Disord. 2008; 23(15): 2129-2170, doi: 10.1002/mds.22340, indexed in Pubmed: 19025984.

6. Martinez-Martin P, Rodriguez-Blazquez C, Alvarez-Sanchez M, et al. Expanded and independent validation of the Movement Disorder Society-Unified Parkinson's Disease Rating Scale (MDS-UPDRS). J Neurol. 2013; 260(1): 228-236, doi: 10.1007/s00415-012-6624-1, indexed in Pubmed: 22865238.

7. Goetz CG, Stebbins GT, Wang Lu, et al. IPMDS-Sponsored Scale Translation Program: Process, Format, and Clinimetric Testing Plan for the MDS-UPDRS and UDysRS. Mov Disord Clin Pract. 2014; 1(2): 97-101, doi: 10.1002/mdc3.12023, indexed in Pubmed: 27747259. 
8. Fowler FJ. Improving Survey Questions: Design and Evaluation. Applied Social Research Methods Series: 38. Thousand Oaks: Sage. : 1995.

9. Muthen LK. and Muthen BO. M plus Version 7 User's Guide. Los Angeles, CA: Muthen. : Muthen.

10. Hatcher L. Step-by-step approach to using the SAS system for factor anaIysis and structural equation modeling. 1994. SAS Inst. , Cary, NC. ; 73.

11. Brown TA. Confirmatory factor analysis for applied research. New York: Guilford SAGE Publications, Inc. : 2006.

12. Browne M. An Overview of Analytic Rotation in Exploratory Factor Analysis. Multivariate Behavioral Research. 2010; 36(1): 111-150, doi: 10.1207/s15327906mbr3601_05.

13. Gorsuch RL. Factor analysis. Second edition. Hillsdale, NJ: Lawrence Erlbaum Associations, Inc. : 1983.
14. Gallagher DA, Goetz CG, Stebbins G, et al. Validation of the MDS-UPDRS Part I for nonmotor symptoms in Parkinson's disease. Mov Disord. 2012; 27(1): 79-83, doi: 10.1002/mds.23939, indexed in Pubmed: 21915909.

15. Goetz CG, Liu Y, Stebbins GT, et al. Gender-, age- and race/ethnicity-based Differential Item Functioning (DIF) analysis of MDS-UPDRS. Mov Disord. 2016; 31(12): 1865-1873.

16. Martínez-Martín P, Rodríguez-Blázquez C, Forjaz MJ, et al. Relationship between the MDS-UPDRS domains and the health-related quality of life of Parkinson's disease patients. Eur J Neurol. 2014; 21(3): 519-524, doi: 10.1111/ene.12349, indexed in Pubmed: 24447695.

17. Balash Y, Korczyn AD, Knaani J, etal. Quality-of-life perception by Parkinson's disease patients and caregivers. Acta Neurol Scand. 2017; 136(2): 151154, doi: 10.1111/ane.12726, indexed in Pubmed: 28083960. 